\begin{tabular}{|c|l|}
\hline Title & $\begin{array}{l}\text { Highly Efficient Room-Temperature Electron-Photon Spin Conversion Using a Semiconductor Hybrid Nanosystem } \\
\text { with Gradual Quantum Dimensionality Reduction }\end{array}$ \\
\hline Author(s) & Hiura, Satoshi; Takishita, Mizuki; Takay ama, Junichi; Sato, Shino; Muray ama, A kihiro \\
\hline Citation & $\begin{array}{l}\text { Physical review applied, 14(4), 044011 } \\
\text { https://doi.org/10.1103/PhysRevA pplied.14.044011 }\end{array}$ \\
\hline Issue Date & 2020-10-08 \\
\hline Doc URL & http://hdl.handle.net/2115/79771 \\
\hline Rights & @2020 A merican Physical Society \\
\hline Type & article \\
\hline File Information & PhysRevA pplied.14.044011.pdf \\
\hline
\end{tabular}

Instructions for use 


\title{
Highly Efficient Room-Temperature Electron-Photon Spin Conversion Using a Semiconductor Hybrid Nanosystem with Gradual Quantum Dimensionality Reduction
}

\author{
Satoshi Hiura $\odot,{ }^{*}$ Mizuki Takishita, Junichi Takayama $\odot$, Shino Sato, and Akihiro Murayama $\odot$ \\ Faculty of Information Science and Technology, Hokkaido University, Kita 14, Nishi 9, Kita-ku, Sapporo \\ 060-0814, Japan
}

(Received 7 July 2020; revised 7 August 2020; accepted 25 August 2020; published 8 October 2020)

\begin{abstract}
Improved electron-photon spin conversion efficiency is a key component of technological platforms for optospintronics integration in information processing; this concept is based on optical devices transmitting and receiving spin information superimposed on light. Semiconductor quantum dots (QDs) are the most promising materials for optospintronic devices; however, in addition to their weak room-temperature luminescence, their electron-photon spin conversion efficiencies are lower than $50 \%$. Here, we present semiconductor QDs embedded in quantum wells (QWs) containing quasi-QDs. The proposed semiconductor hybrid nanosystem with gradual quantum dimensionality reduction demonstrates luminescence one order of magnitude stronger than that of conventional QDs and an electron-photon spin conversion efficiency of almost $80 \%$ at room temperature. Optical characterization reveals that efficient carrier capture, suppressed depolarized-spin reinjection, and quasi-three-dimensional quantum confinements in the QWs facilitate the highly efficient electron-photon spin conversion. This study constitutes a significant advance towards the realization of QD-based spin-functional optical devices for electron-spin-based quantum information platforms.
\end{abstract}

DOI: 10.1103/PhysRevApplied.14.044011

\section{INTRODUCTION}

Data traffic is increasing exponentially on a yearly basis as a result of recent developments in cloud computing $[1,2]$, the Internet of Things [3,4], and artificial intelligence $[5,6]$. This trend is problematic, however, as power consumption associated with data transmission continues to increase explosively. In current information processing and optical communication technology, electrons and photons are utilized as information carriers $[7,8]$. The spins of these carriers are used as information memory and generate no electric power consumption. Therefore, ultra-low powerconsumption electronic circuits can be realized by utilizing spin for electronic information processing; this is known as spintronics [9-11].

To date, there has been much breakthrough in metalbased spintronics, such as magnetic data storage and memory applications through giant and tunnel magnetoresistance in layered magnetic structures $[12,13]$. In contrast, utilization of semiconductor spintronics promises more versatile applications, such as optical spin devices $[14,15]$ and better integrability with traditional semiconductor technologies than metal-based spintronics $[16,17]$. Semiconductor spintronics can also bridge next-generation

\footnotetext{
*hiura@ist.hokudai.ac.jp
}

spintronics-based information processing and optical interconnection by photoelectric spin conversion, which is a key technology toward an on-demand transmission of large amounts of information. However, in the semiconductor quantum wells (QWs) used for optical devices, rapid spin relaxation at room temperature is unavoidable $[18,19]$; this yields spin information loss, i.e., low electronphoton spin conversion efficiency. Therefore, there is a strong demand for light sources that can retain spin information during light emission at room temperature. Group III-V semiconductor quantum dots (QDs) are known to be the most promising materials for the light sources because of the excellent optical properties [20] and the suppressed carrier spin relaxation [21-25] obtained via their three-dimensional quantum confinements. Up to now, time-resolved optical spin orientation measurements in (In, Ga)As QDs have been performed to study the temperature dependence of the electron-spin relaxation mechanism in QDs [26-29]. A recent study of (In, Ga)As QD ensembles revealed that at temperatures above $140 \mathrm{~K}$, the electron-spin relaxation can be accelerated by the reinjection of depolarized electron spins from the adjacent barriers after a thermal escape from the QDs [28]. A $p$-doped capping barrier was found to suppress the relaxation of the reinjected electron spins due to the weakened D'yakonovPerel' (DP) spin relaxation in the barrier, leading to the 
enhanced electron-photon spin conversion efficiency of QDs [29]. However, the room-temperature luminescence intensity of the QDs was extremely weak as a result of the dominant thermal escape of carriers. Therefore, an appropriate design of the quantum structure surrounding the QDs, such as the barrier height and quantum confinements, is necessary for the room-temperature operation of optical spin devices.

The QD-in-QW (DIW) structure has demonstrated superior optical properties to conventional QDs because the QW promotes the capture of carriers into QDs and suppresses the thermal escape of carriers out of QDs at high temperatures [30,31]. Because of these characteristics, the thermal quenching of QD luminescence, which becomes dominant at temperatures above $200 \mathrm{~K}$ [32], can be greatly suppressed. For spin-related properties, the DIW structure may improve the electron-photon spin conversion efficiency of QDs. Since the conversion efficiency depends on the electron-spin relaxation in the layers surrounding the QDs [28], the DIW structure has the potential to realize a high performance due to the better spin conservation in the QW compared to the bulk. However, there remains a problem to be solved toward highly efficient conversion at room temperature. Note that the electron-spin relaxation time of the $\mathrm{GaAs} /(\mathrm{Al}, \mathrm{Ga}) \mathrm{As} \mathrm{QW}$ is known to be as short as several tens of picoseconds at room temperature due to the dominant DP spin relaxation [18], which is one order of magnitude shorter than the radiative lifetime of QDs [33]. This value is almost the same as the electronspin relaxation time of 42 ps for the GaAs bulk [34]. Therefore, toward the practical application of QD-based optical spin devices, it is highly required to embed the QDs in a nanostructure with a stronger quantum confinement than the QW to suppress the DP spin relaxation, which degrades the room-temperature electron-photon spin conversion efficiency.

In this study, we propose a concept of a DIW-based nanostructure, referred to as QD-in-quasi-QD. For an (In, Ga)As/GaAs/(Al, Ga)As DIW structure with a thin QW, an (In, Ga)As quasi-QD is found to be created within a GaAs QW as a result of the wide In distribution around the (In, Ga)As QDs. This semiconductor hybrid nanosystem with gradual quantum dimensionality reduction exhibits luminescence that is one order of magnitude stronger than that of conventional QDs, along with an electron-photon spin conversion efficiency of almost $80 \%$ at room temperature compared to $50 \%$ for conventional QDs. This higher conversion efficiency is mainly derived from the suppressed relaxation of electron spins reinjected from the QW containing quasi-QD, where the electronspin relaxation time is as long as approximately 200 ps due to the quasi-three-dimensional quantum confinements. This study is also the exploration of the electron-spin properties in the DIW structure, and provides a comprehensive understanding of the mechanism of the obtained high performance by investigating the electron-spin dynamics ranging from 6 to $293 \mathrm{~K}$.

\section{EXPERIMENTAL DETAILS}

The self-assembled $\mathrm{In}_{0.5} \mathrm{Ga}_{0.5} \mathrm{As} / \mathrm{GaAs}$ QDs and the $\mathrm{In}_{0.5} \mathrm{Ga}_{0.5} \mathrm{As} / \mathrm{GaAs} / \mathrm{Al}_{0.15} \mathrm{Ga}_{0.85} \mathrm{As}$ DIW samples are grown using an EIKO molecular beam epitaxy system on semiinsulating $\operatorname{GaAs}(001)$ substrates with a 400-nm-thick GaAs buffer. Two types of DIW sample are studied: $\mathrm{Al}_{0.15} \mathrm{Ga}_{0.85} \mathrm{As} / \mathrm{GaAs} / \mathrm{In}_{0.5} \mathrm{Ga}_{0.5} \mathrm{As} \quad \mathrm{QD} / \mathrm{GaAs} / \mathrm{Al}_{0.15} \mathrm{Ga}_{0.85}$ As 30/16/2.1/14/30 and 30/9/2.1/6/30 nm, hereafter labeled as DIW-30 nm and DIW-15 nm, respectively. The $\mathrm{In}_{0.5} \mathrm{Ga}_{0.5} \mathrm{As}$ QDs and the GaAs cap layer are grown at $773 \mathrm{~K}$, and all other layers are grown at $853 \mathrm{~K}$. Reference $\mathrm{In}_{0.5} \mathrm{Ga}_{0.5}$ As QDs are prepared under the same growth conditions, and additional QDs are grown on the cap layer for structural characterization via atomic force microscopy (AFM). The AFM analysis of QD structures revealed an areal density of $1.3 \times 10^{10} \mathrm{~cm}^{-2}$ with a 22$\mathrm{nm}$ average diameter (see the Supplemental Material [35]). Scanning transmission electron microscopy (STEM) samples are prepared via a standard bulk-pick-up method using a focused ion beam instrument. The STEM analysis is performed using an aberration-corrected FEI-Titan Cubed electron microscope equipped with an energy-dispersive $\mathrm{x}$ ray (EDX) detector and operated at $300 \mathrm{kV}$. High-angle annular dark-field STEM (HAADF STEM) and brightfield STEM (BF STEM) imaging are utilized in combination to image the nanostructure surrounding the QDs. In addition, EDX analysis is used to map the atomic species inside and around the QDs.

The circularly polarized photoluminescence (PL) and its time-resolved spectra are measured using a streak camera combined with a spectrometer under circularly $\left(\sigma^{+}\right)$ polarized excitation at 6-293 K. Both the excitation and detection directions coincided with the sample growth direction. A mode-locked Ti:sapphire pulsed laser with an $80-\mathrm{MHz}$ repetition rate, less than 100 -fs pulse width, and $10-\mathrm{nm}$ spectral width is used as the excitation source. The excitation laser spot diameter is approximately $0.1 \mathrm{~mm}$. The full width at half maximum of the laser-pulse time response curve is 10 ps. For polarization-resolved optical characterization, a linear polarizer and quarter-wave plate are inserted into the excitation (detection) beam paths to generate (detect) circularly polarized excitation (emission). For PL measurements of the reference QD and DIW samples, the excitation energies are tuned to 780 $\mathrm{nm}(1.59 \mathrm{eV})$ and $700 \mathrm{~nm}(1.77 \mathrm{eV})$ at $6 \mathrm{~K}$, which is above the band gap of the GaAs and $\mathrm{Al}_{0.15} \mathrm{Ga}_{0.85}$ As barrier. These excitation energies are changed in a stepwise manner with increasing temperature and according to the band-gap shrinkage. The excitation powers of the reference QD, DIW-30 nm, and DIW-15 nm are 0.85, 2.5, and $5 \mathrm{~mW}$, respectively. Under this excitation condition, the 
integrated QD PL intensities, which corresponded to the number of carriers injected into the QDs, are almost identical at $6 \mathrm{~K}$. The electron-spin polarization $\left(P_{e}\right)$ generated in the GaAs or in the $\mathrm{Al}_{0.15} \mathrm{Ga}_{0.85} \mathrm{As}$ barrier is expected to be $50 \%$, based on the optical-transition selection rule [36]. Here, the PL circular polarization degree (CPD) is defined as $\mathrm{CPD}=\left(I_{\sigma+}-I_{\sigma-}\right) /\left(I_{\sigma+}+I_{\sigma-}\right)$, where $I_{\sigma \pm}$ denotes the $\sigma^{ \pm}$-polarized PL intensity. The CPD measured in QDs reflects $P_{e}$, because the hole spins are rapidly depolarized in a barrier with a spin relaxation time of less than $1 \mathrm{ps}$ [37,38], and QD PL mainly involves the heavy-hole state, because of the large splitting between the heavy-hole and light-hole states in (In, Ga)As QDs [39].

\section{RESULTS AND DISCUSSION}

During formation of a DIW structure, an intermediate structure of inhomogeneous composition is expected to exist [30,31]. Therefore, we performed cross-sectional HAADF STEM and EDX analysis to explore the intermediate structure formed within the GaAs QW, as shown in Figs. 1(a)-1(f). The In atoms are found to be widely distributed in the stacking direction around the QDs. The In distribution is also confirmed from BF STEM images, in which dark regions mainly indicated the presence of In atoms (see the Supplemental Material [35]). The reference QD exhibited the truncated pyramidal shape characteristic of (In, Ga)As QDs [40]; however, this shape became close to ellipsoidal as the QD base length is retained but the QW thickness is decreased. These results indicate the formation of intermediate (In, Ga)As layers within the GaAs QW particularly for DIW-15 nm, as illustrated in Fig. 1(g).

The realized DIW structure can provide three advantages for electron-photon spin conversion at high temperatures: (i) efficient capture of carriers into QDs [30,31];
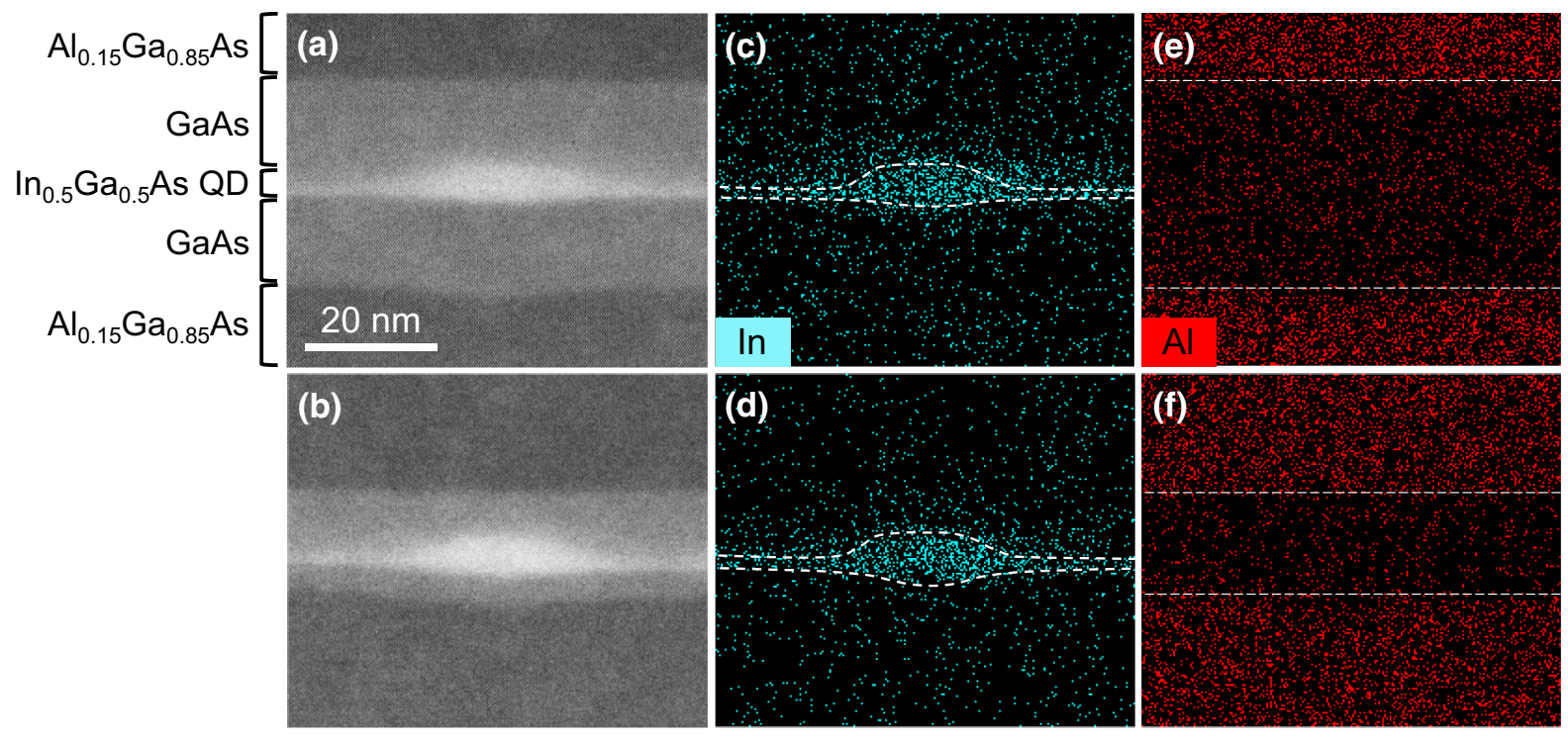

(g)
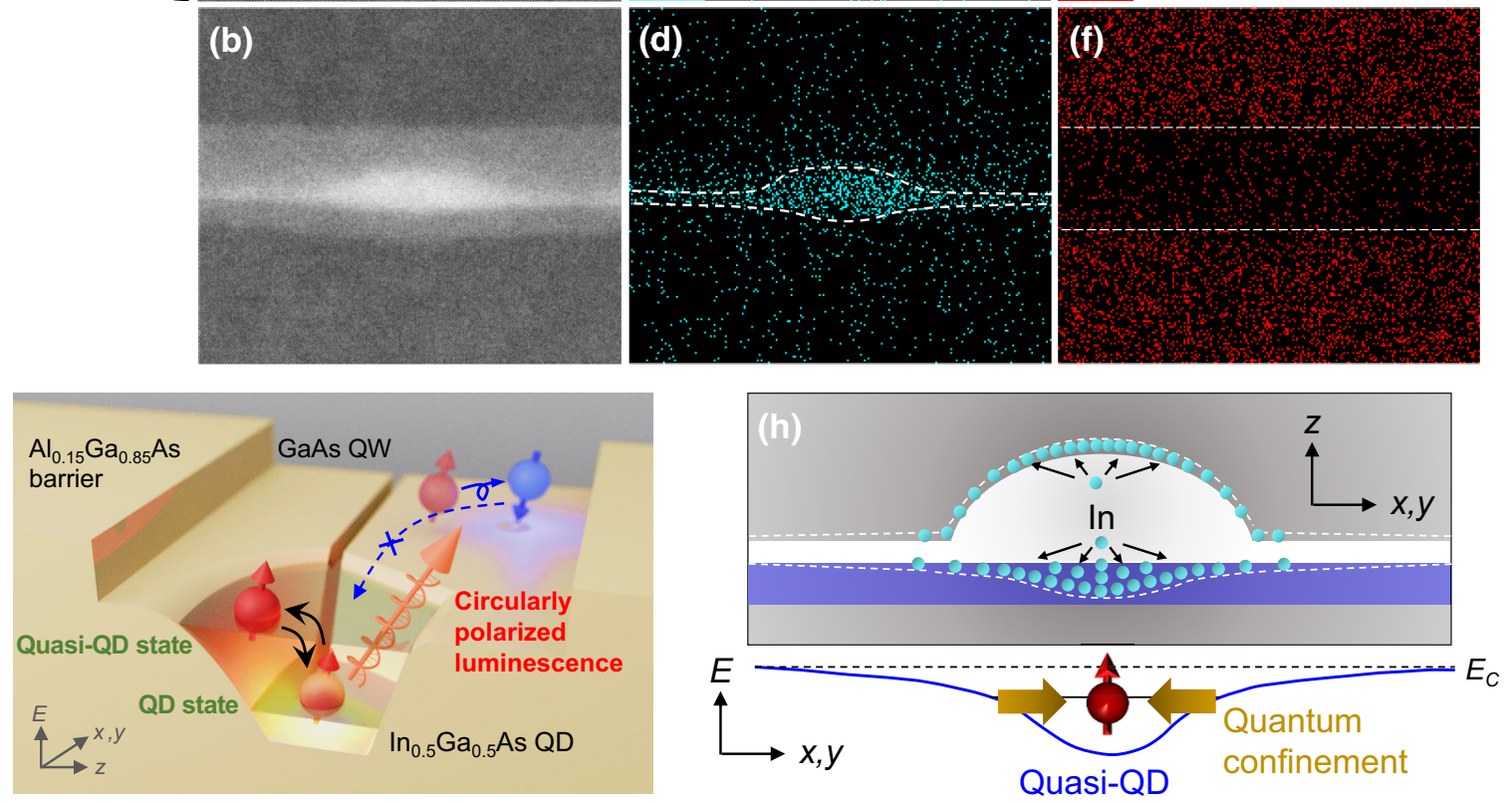

FIG. 1. Structural characterization of the DIW containing quasi-QD. (a),(b) HAADF STEM images and EDX elemental maps of (c),(d) In and (e),(f) Al for DIW-30 nm and DIW-15 nm, respectively. (g) Schematic illustration of the conduction band profile in the semiconductor hybrid nanosystem with gradual quantum dimensionality reduction. The highly efficient electron-photon spin conversion achieved in the proposed nanosystem is attributed to blocked reinjection of the depolarized spins from the adjacent QW (blue dashed arrow) due to efficient radiative recombination, along with suppressed relaxation of the reinjected electron spins (black solid arrow) due to the quasi-three-dimensional quantum confinements at the quasi-QD state. (h) Schematic illustration of the DIW structure indicating wide In-atom distribution around QDs. An intermediate (In, Ga)As layer corresponding to a quasi-QD with quasi-three-dimensional quantum confinements is formed within the GaAs QW. 
(ii) suppressed reinjection of depolarized spins due to efficient radiative recombination in the QW (this originates from stronger overlap of the carrier wave functions compared to that of the bulk material); and (iii) suppressed relaxation of reinjected electron spins owing to the quantum confinement in the QW. In this study, an intermediate layer having a quasi-zero-dimensional electronic nature is formed within the QW, as illustrated in Fig. 1(h). The broad distribution of the In atoms around the QD creates a deep energy potential within the GaAs QW, yielding both lateral and vertical quantum confinements. Therefore, the intermediate layer behaves as a quasi-QD.

In Figs. 2(a)-2(c) we show contour maps of the temperature-dependent PL intensity as a function of photon energy. The PL emission is from the wide energy regions including the QD ground state (GS) and excited states (ESs). No substantial difference in the QD GS is observed for the different samples, although the QD GS of DIW-30 nm is slightly shifted towards a higher energy (see the Supplemental Material [35]); this is likely due to the lower In composition of the QDs in that sample. In Fig. 2(d) we show the PL peak energy as a function of temperature. At temperatures below $100 \mathrm{~K}$, the emission energy followed the band-gap temperature dependencies obtained by Varshni's law with the appropriate parameter for $\operatorname{In}_{0.5} \mathrm{Ga}_{0.5} \mathrm{As}$ [41]. However, the emission energy decreased with increasing temperature above $100 \mathrm{~K}$, at a rate faster than that given by Varshni's law. This fast redshift can be explained by carrier activation and transfer from higher- to lower-energy QDs via the GaAs layers [42]. The peak energy is constantly lower than that given by Varshni's law for the reference $\mathrm{In}_{0.5} \mathrm{Ga}_{0.5}$ As QD. However, the redshift decelerated for the DIW samples. Note that the emission energy became constant at temperatures above $160 \mathrm{~K}$ for DIW-15 nm. With increasing temperature above $150 \mathrm{~K}$, the PL properties are dominated by the electron-phonon scattering; this yielded a monotonic increase in the PL linewidth (see the Supplemental Material [35]) and a lower emission energy decrease rate [43]. These findings indicate termination of the electron (a) Reference QD
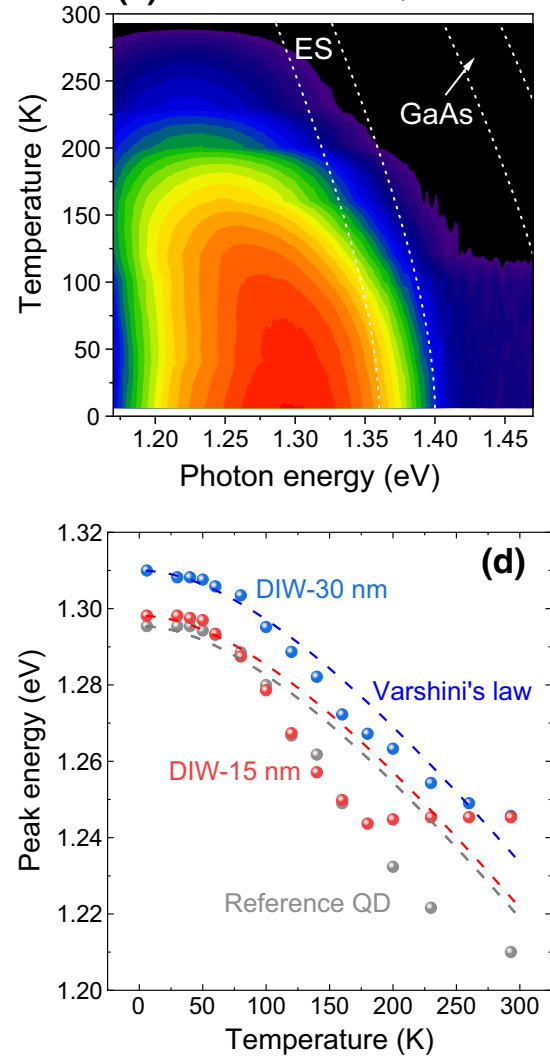

(b) DIW-30 nm
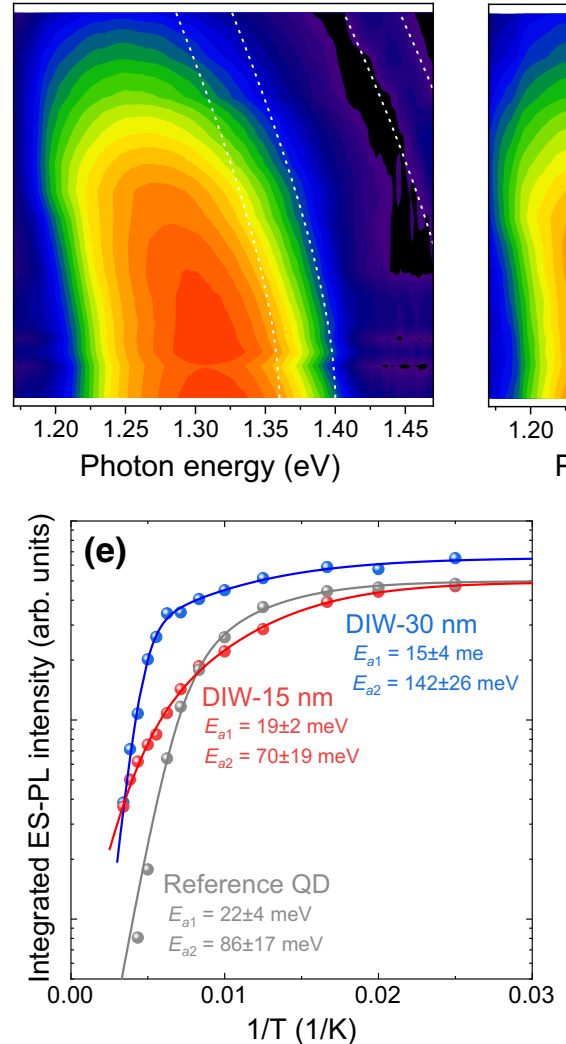

(c) DIW-15 nm PL intensity (arb. units)
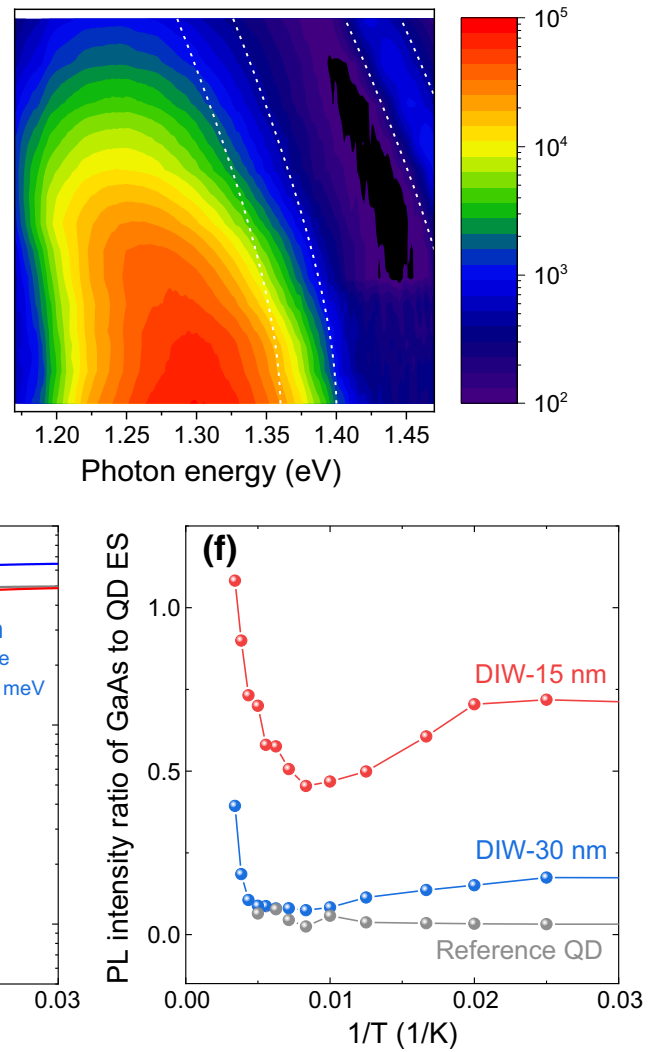

FIG. 2. Contour maps of the temperature-dependent PL intensity as a function of photon energy for (a) the reference QD, (b) DIW$30 \mathrm{~nm}$, and (c) DIW-15 nm. The energy regions surrounded by the white dashed lines indicate the $\operatorname{In}_{0.5} \mathrm{Ga}_{0.5} \mathrm{As}$ QD ES focused on in this study and the GaAs barrier or QW states, which are shifted with changing temperature in accordance with Varshni's law with the appropriate parameter for $\mathrm{In}_{0.5} \mathrm{Ga}_{0.5} \mathrm{As}$ and GaAs [41]. (d) PL peak energies with the predicted temperature-dependent energy shift by Varshni's law (dashed lines), (e) integrated PL intensities of QD ESs with Arrhenius fits (solid lines), and (f) PL intensity ratios of GaAs to QD ES as functions of temperature. For the reference QD, the PL intensity ratio at temperatures above $200 \mathrm{~K}$ is not plotted because the PL intensity is weak under those conditions. 
thermal redistributions via carrier activation and transfer at the lowest temperatures for DIW-15 nm; i.e., less thermal energy is required for electron delocalization due to the formation of intermediate ( $\mathrm{In}, \mathrm{Ga}$ )As layers within the GaAs QW. Hence, the electron-phonon scattering became more dominant at high temperatures for DIW-15 nm, yielding a constant emission energy. This behavior is observed at higher temperatures for DIW-30 nm.

As shown in Fig. 2(a), dramatic PL quenching appeared at approximately $200 \mathrm{~K}$ for the reference QD, where the QD luminescence at room temperature became two orders of magnitude weaker than that at $160 \mathrm{~K}$. This result is in good agreement with a previous finding for InAs/GaAs QDs [32]. The results for the DIW samples, however, indicate a sharp contrast, as PL quenching is significantly suppressed [see Figs. 2(b) and 2(c)]. In Fig. 2(e) we show the integrated PL intensity of the QD ESs as a function of temperature, where the QD ES is defined as the energy range of $1.36-1.40 \mathrm{eV}$ at $6 \mathrm{~K}$. The analyzed QD ES energy range shifted with changing temperature in accordance with Varshni's law. Two activation-energy components are apparent. The first component of approximately 20 meV corresponds to the energy difference between the QD ESs based on the three-dimensional quantum simulations, where the QD diameter determined by AFM analysis [44] and the nonuniform In composition inside the QD [45] are taken into account (see the Supplemental Material [35]); these simulations are performed using the nextnano commercial software [46]. Thermal excitation of the electrons induced PL quenching due to the decrease in the radiative recombination efficiency, which is attributed to the weakened overlap of the electron and hole wave functions. Note that, for the reference QD, the second activation energy of $86 \mathrm{meV}$ is related to electrons escaping from the QD ESs to nonradiative recombination centers through the GaAs barriers $[42,47]$. For DIW-30 nm, the activation energy is much larger at $142 \mathrm{meV}$, and is within the range of the calculated energy difference between the QD ES and the $\mathrm{Al}_{0.15} \mathrm{Ga}_{0.85}$ barrier (see the Supplemental Material [35]). This result indicates weakened thermal escape from the QDs, i.e., thermally escaped electrons are reinjected from the QWs to the QDs and subsequently recombined with the holes. It should be noted that the second component of DIW-15 nm is only $70 \mathrm{meV}$, smaller than that of the reference QD. The thermal excitation to the intermediate (In, Ga)As layer within the GaAs QW and the subsequent radiative recombination are responsible for the smallest activation energy, as explained below.

We observed PL emission from the GaAs QWs for the DIW samples at $6 \mathrm{~K}$ (see the Supplemental Material [35]). The GaAs PL intensity of DIW-15 nm is four times stronger than that of DIW-30 nm, demonstrating the higher radiative recombination efficiency in that QW. In Fig. 2(f) we show the ratio of the GaAs PL intensities to those of the QD ESs as a function of temperature. For the reference
QD, the ratio is independent of temperature. For DIW-30 $\mathrm{nm}$ and DIW-15 nm, the ratio increased dramatically with increasing temperature above 200 and $120 \mathrm{~K}$, respectively. Note that the GaAs PL intensity became comparable to that of the QD ES at $293 \mathrm{~K}$ for DIW-15 nm. The thermally escaped electrons tended to recombine with the holes in the QW because of the high recombination efficiency. Hence, the reinjection of thermally escaped carriers is significantly suppressed for DIW-15 nm.

Next, to explore the electron-photon spin conversion efficiency, spin-polarized light emission properties are studied. Generally, $P_{e}$ in a semiconductor can be described in the simplest form by the rate equation [36]

$$
P_{e}=\frac{P_{0}}{1+\tau_{r} / \tau_{s}} .
$$

Here, $\tau_{r}$ and $\tau_{s}$ denote the radiative lifetime and electronspin relaxation time, respectively, and $P_{0}$ is the initial electron-spin polarization during injection into emissive states, which depends on the degree of electron-spin relaxation during injection. In this study, the term $\left(1+\tau_{r} / \tau_{s}\right)^{-1}$ is referred to as the net electron-photon spin conversion efficiency of QDs.

In Figs. 3(a)-3(c) we show contour maps of the temperature-dependent CPD of the PL as a function of the photon energy for the examined samples. The CPD measured in the QDs reflects $P_{e}$, as explained above. At $6 \mathrm{~K}$, we observed the maximum $P_{e}$ of $60 \%-70 \%$ for the QD ES (see the Supplemental Material [35]); this is higher than the initial polarization of $50 \%$ optically generated in the barrier. This $P_{e}$ amplification is due to selective relaxation of the minority spins from the QD ESs to the QD GS relative to the blocked relaxation of the majority spins due to Pauli blocking [48]. In Fig. 3(d) we show the averaged $P_{e}$ of the QD ES as a function of temperature. The $P_{e}$ values of the reference QD and DIW-30 nm decreased dramatically with increasing temperature above $50 \mathrm{~K}$ and became only $5 \%-10 \%$ above $200 \mathrm{~K}$. In contrast, a $P_{e}$ of $20-30 \%$ accompanied by strong luminescence is obtained for the wide energy region of DIW-15 nm at temperatures above $200 \mathrm{~K}$, as shown in Figs. 3(c) and 3(d). In Fig. 3(e) we show the circularly polarized time-integrated PL spectra and the corresponding $P_{e}$ measured at $293 \mathrm{~K}$. The $P_{e}$ of DIW-30 nm is slightly higher than that of the reference QD $(230 \mathrm{~K})$, whereas that of DIW-15 nm more than doubled. We also found that the GaAs $P_{e}$ reached $10 \%$ for DIW$15 \mathrm{~nm}$ compared to $0 \%$ for the reference QD. This result clearly indicates significant suppression of electron-spin relaxation in the GaAs QW.

To quantitatively reveal the origin of the enhanced $P_{e}$, we performed a time-resolved analysis of the PL and $P_{e}$ of the QD ESs. The circularly polarized PL time profiles and the corresponding $P_{e}$, measured at $293 \mathrm{~K}$, are shown in Fig. 4(a). Here, the $P_{e}$ decay time constant corresponds to half 


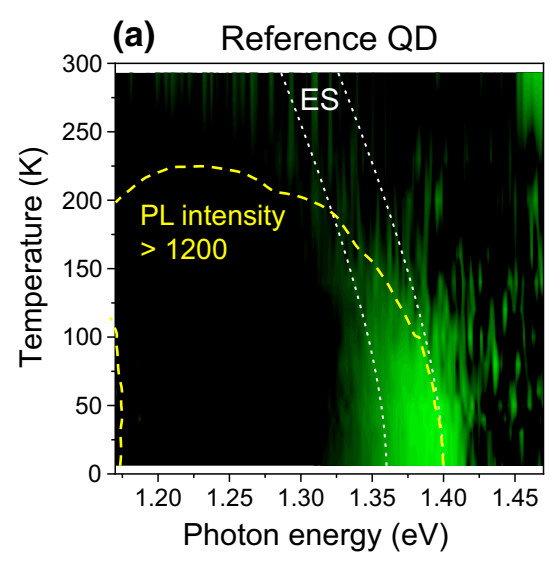

(b) DIW-30 nm

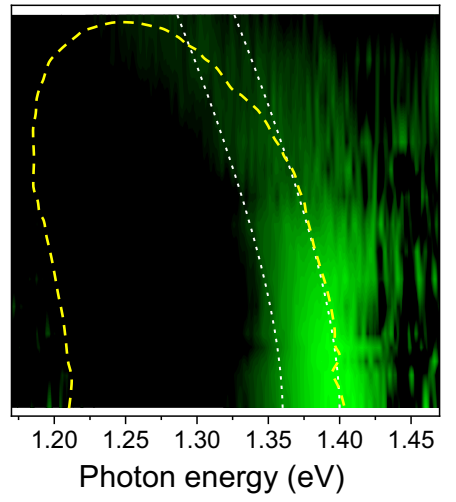

(c) $\quad$ DIW-15 nm $\quad \mathrm{CPD}=P_{e}(\%)$

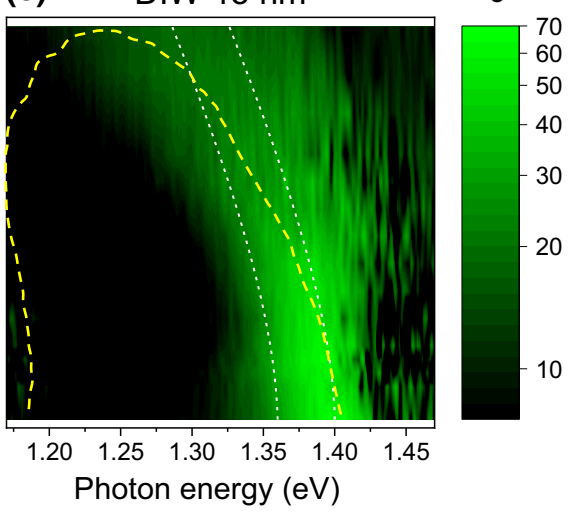

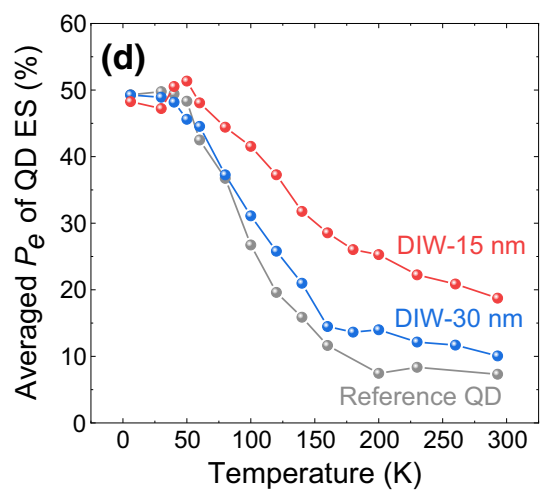
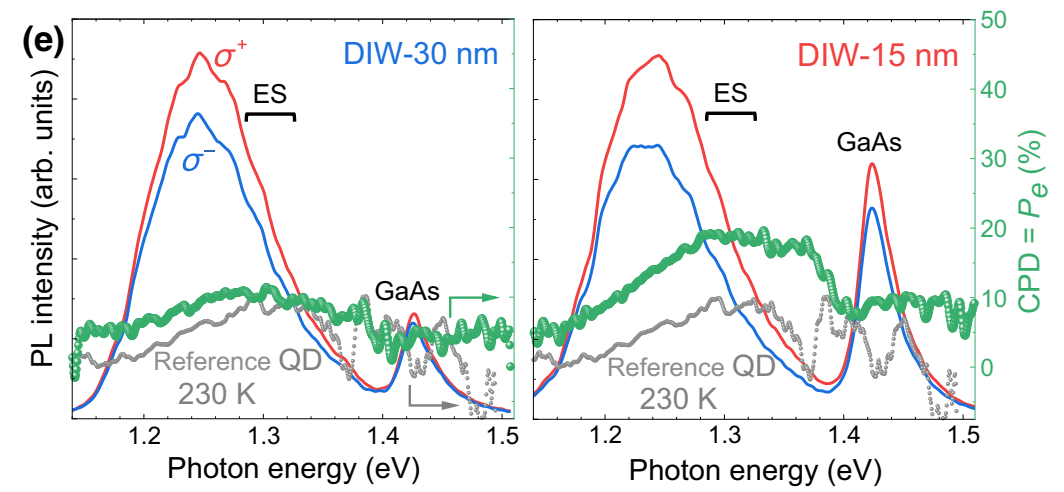

FIG. 3. Contour maps of temperature-dependent $P_{e}$ as a function of photon energy for (a) the reference QD, (b) DIW-30 nm, and (c) DIW-15 nm. The energy regions surrounded by the white dashed lines indicate the $\operatorname{In}_{0.5} \mathrm{Ga}_{0.5} \mathrm{As}$ QD ES focused on in this study, which is shifted with changing temperature in accordance with Varshni's law with the appropriate parameter for $\mathrm{In}_{0.5} \mathrm{Ga}_{0.5} \mathrm{As}[41$ ]. The areas surrounded by the yellow dashed lines show the temperature and energy ranges where the PL intensities larger than 1200 are obtained in the PL intensity maps of Figs. 2(a)-2(c). (d) Averaged $P_{e}$ values of QD ESs as functions of temperature. (e) Circularly polarized time-integrated PL spectra and corresponding $P_{e}$ values of DIW-30 nm (left) and DIW-15 nm (right), measured at $293 \mathrm{~K}$. For comparison, the corresponding $P_{e}$ of the reference QD measured at $230 \mathrm{~K}$ is indicated by the gray symbols.

the electron-spin relaxation time constant in a conventional spin-split two-level system [26]. The resulting $\tau_{s}$ for DIW$30 \mathrm{~nm}$ is $202 \mathrm{ps}$, compared to $140 \mathrm{ps}$ for the reference QD $(230 \mathrm{~K})$. For DIW-15 nm, $\tau_{s}$ is further increased to 307 ps. This value is one order of magnitude longer than the room-temperature value for the $\mathrm{GaAs} /(\mathrm{Al}, \mathrm{Ga}) \mathrm{As} \mathrm{QW}$ of $32 \mathrm{ps}[18]$.

We also found a large difference in $\tau_{r}$ between the DIW samples. For simplicity, $\tau_{r}$ is defined as the decay time constant of the sum of $\sigma^{ \pm}$-polarized PL intensity. In Fig. 4(b) we show $\tau_{r}$ as a function of temperature for DIW-30 nm and DIW-15 nm. For the former, $\tau_{r}$ gradually increased from $200 \mathrm{ps}$ at $60 \mathrm{~K}$ to $302 \mathrm{ps}$ at $200 \mathrm{~K}$. The increased $\tau_{r}$ indicates weakened thermal escape from the QDs, i.e., dominant reinjection of electron spins from the QWs. As a result, the PL quenching is significantly suppressed in this temperature regime, as shown in Figs. 2(b) and 2(e). With further increasing temperature, $\tau_{r}$ decreased dramatically to $164 \mathrm{ps}$ at $293 \mathrm{~K}$ as a result of the increasing thermal escape. The behavior for DIW-15 nm exhibited a sharp contrast, as $\tau_{r}$ gradually decreased from $192 \mathrm{ps}$ at $6 \mathrm{~K}$ to $93 \mathrm{ps}$ at $293 \mathrm{~K}$. No increase in $\tau_{r}$ is observed, which clearly demonstrates significant reinjection suppression. Regardless of the shortened radiative lifetime, strong QD PL emission comparable to that of DIW-30 nm appeared at 293 K, as shown in Figs. 2(c) and 2(e). This highly efficient light emission is derived from the more efficient carrier capture. At temperatures above $200 \mathrm{~K}$, the PL intensity of the QD ES integrated in the initial time region of $0-30 \mathrm{ps}$ for DIW-15 nm is approximately double that for DIW-30 $\mathrm{nm}$ (see the Supplemental Material [35]).

With increasing temperature, $\tau_{s}$ is dominated by the DP spin relaxation mechanism and decreases according to a $T^{-3}$ and $T^{-1}$ dependence in the bulk material and QW, respectively [19]. Therefore, the quantum confinement dimensionality can be determined by the temperature dependence of $\tau_{s}$. In Fig. 4(c) we show the $\tau_{s}$ values of the QD ESs as functions of temperature. For the reference $\mathrm{QD}, \tau_{s}$ began to decrease above $50 \mathrm{~K}$ and exhibited a $T^{-1.8}$ dependence. The observed $\tau_{s}$ included the electron-spin 

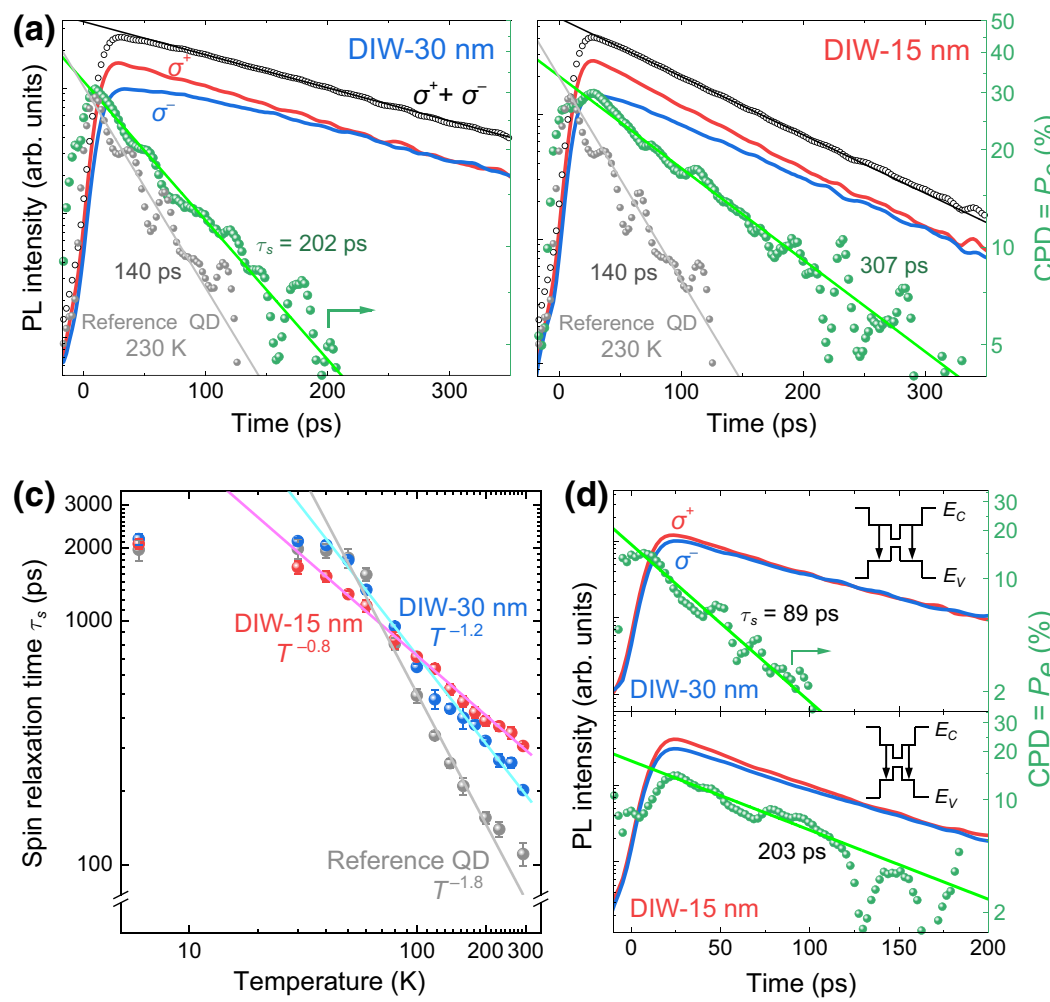
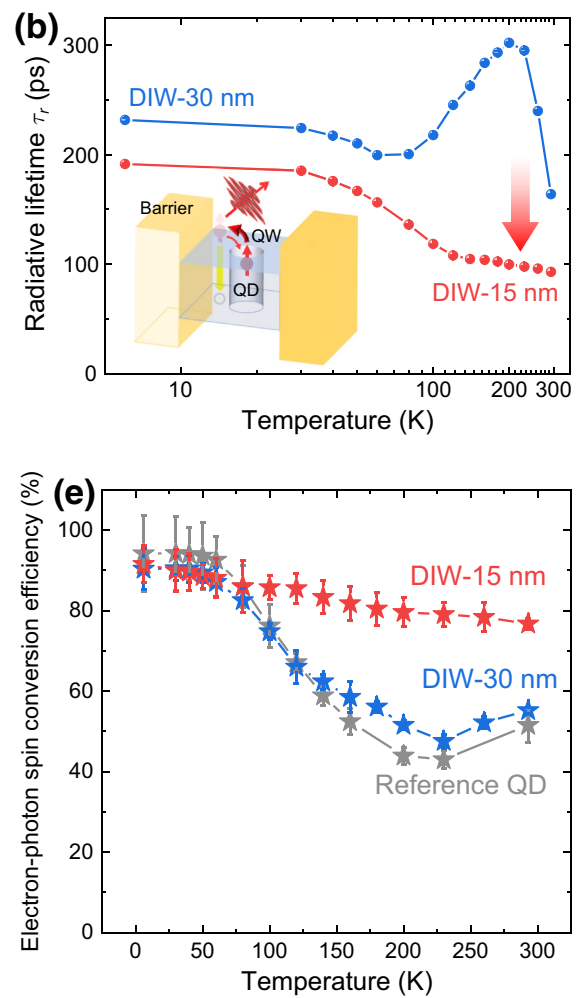

FIG. 4. (a) Circularly polarized PL time profiles and corresponding $P_{e}$ values of QD ESs for DIW-30 nm (left) and DIW-15 nm (right), measured at $293 \mathrm{~K}$. The sum of $\sigma^{ \pm}$-polarized PL intensity as a function of time with the single-exponential decay fittings (black solid lines) is also shown. For comparison, the corresponding $P_{e}$ of the reference QD measured at $230 \mathrm{~K}$ is indicated by the gray symbols. The gray and green solid lines show the single-exponential decay fittings for the time-dependent CPD. (b) Radiative lifetimes (decay time constant of the sum of $\sigma^{ \pm}$-polarized PL intensity) and (c) electron-spin relaxation times as functions of temperature. The inset in (b) shows the suppressed reinjection of electron spins from the QW due to the efficient radiative recombination there. The gray, cyan, and magenta solid lines in (c) show the $T^{-1.8}, T^{-1.2}$, and $T^{-0.8}$ dependencies, respectively. (d) Circularly polarized PL time profiles and corresponding $P_{e}$ values of the GaAs QW, measured at $293 \mathrm{~K}$. The green solid lines show the single-exponential decay fittings for the time-dependent CPD. (e) Electron-photon spin conversion efficiencies as functions of temperature.

relaxation in both the QDs and the barrier. The reinjection of depolarized electron spins from the barrier largely decreased $\tau_{s}$ with increasing temperature [28].

This decrease in $\tau_{s}$ could be significantly mitigated by introducing the DIW structure. For DIW-30 nm, $\tau_{s}$ exhibited a $T^{-1.2}$ dependence, which is close to the DP spin relaxation in the QW. This behavior can be interpreted by considering dominant reinjection of the electron spins from the QW at high temperatures, as discussed above. It should be noted that the $\tau_{s}$ of DIW-15 nm exhibited a $T^{-0.8}$ dependence. This result is closer to that for an ideal QD, in which the DP spin relaxation is negligible. We expect that the thinner QW suppressed the relaxation of the reinjected electron spins, yielding a slower $P_{e}$ decay during light emission. This finding is supported by the large increase in $\tau_{s}$ in the QW, as shown in Fig. 4(d). A much longer $\tau_{s}$ is obtained for DIW-15 nm compared to that for DIW-30 nm, at 203 and $89 \mathrm{ps,} \mathrm{respectively.} \mathrm{This} \mathrm{result}$ demonstrates that the thinner QW containing quasi-QD examined in this study has strong quantum confinements comparable to the QD, i.e., quasi-three-dimensional quantum

confinements.

In Fig. 4(e) we show the electron-photon spin conversion efficiencies as functions of temperature, which are obtained using $\tau_{r}$ and $\tau_{s}$ based on Eq. (1). It should be noted that the DIW-15-nm conversion efficiency reached almost $80 \%$ at temperatures above $200 \mathrm{~K}$. This value is approximately double that for the reference QD and DIW$30 \mathrm{~nm}$. Here, the highest $P_{e}$ up to $35 \%$ at room temperature has been previously reported in InAs/GaAs QDs under resonant circularly polarized excitation from the wettinglayer heavy-hole band (i.e., complete spin polarization of photogenerated carriers) [32]. The absolute $P_{e}$ value is higher than that achieved in this study, while the conversion efficiency has been limited to $35 \%$, assuming that the electron-spin relaxation during ultrafast injection into the QD emissive state is negligible. In contrast, the timeaveraged $P_{e}$ for DIW-15 nm largely decreased from 50\% at $6 \mathrm{~K}$ to $20 \%$ at $293 \mathrm{~K}$ [see Fig. 3(d)]. This decrease in 
$P_{e}$ is mainly caused by the decrease in the initial $P_{e}$ with increasing temperature (see the Supplemental Material [35]), which is due to the dominant DP spin relaxation in the three-dimensional barriers. Therefore, the net electronphoton spin conversion efficiency of the QDs is shown in Fig. 4(e). The superior conversion efficiency is attributed to the combination of the suppressed reinjection of the electron spins (decreased $\tau_{r}$ ) and the suppressed relaxation of the electron spins reinjected from the QW (increased $\tau_{s}$ ) (see the Supplemental Material [35]). These findings indicate that suppression of the electron-spin relaxation in the layers surrounding the QDs is the important factor for realization of highly efficient electron-photon spin conversion at room temperature, and demonstrate that the proposed semiconductor hybrid nanosystem is the promising light source for optical spin devices operating at room temperature.

\section{CONCLUSION}

In conclusion, we have demonstrated an electron-photon spin conversion efficiency of almost $80 \%$ at room temperature using (In, Ga)As QDs embedded in a GaAs QW, in which the wide distribution of the In atoms around the QDs creates a quasi-QD with quasi-three-dimensional quantum confinements. This semiconductor hybrid nanosystem with gradual quantum dimensionality reduction achieves efficient carrier capture and yields room-temperature luminescence stronger than that for conventional QDs by more than one order of magnitude. We have also demonstrated an electron-spin relaxation time that is more than three times longer than the radiative lifetime. The highly efficient electron-photon spin conversion achieved in this study is attributed to suppressed reinjection of the depolarized spins from the adjacent QW, along with suppressed relaxation of the electron spins reinjected from the QW due to the quasi-three-dimensional quantum confinements in the QW containing quasi-QD. Therefore, the findings of this study suggest that suppression of thermally excited electron-spin relaxation around QDs is crucial for future room-temperature operation of QD-based spin-functional optical devices. The present semiconductor hybrid nanosystem paves the way for the development of a self-assembled dot-in-dot structure, which can further enhance the room-temperature electron-photon spin conversion efficiency.

\section{ACKNOWLEDGMENTS}

This work is supported by the Japan Society for the Promotion of Science (JSPS) under Grants No. 16H06359, No. 19K15380, and No. 19H05507. This work is also supported by the Hattori Hokokai Foundation. This work is also partly supported by the Hokkaido University microstructural characterization platform as a program of the Nanotechnology Platform of the Ministry of Education,
Culture, Sports, Science and Technology (MEXT), Japan, Grant No. PJPMXP09A19HK0064.

[1] M. Armbrust, A. Fox, R. Griffith, A. D. Joseph, R. Katz, A. Konwinski, G. Lee, D. Patterson, A. Rabkin, I. Stoica, and M. Zaharia, A view of cloud computing, Commun. ACM 53, 50 (2010).

[2] A. Fox, Cloud computing - What's in it for me as a scientist?, Science 331, 406 (2011).

[3] R. Haight, W. Haensch, and D. Friedman, Solar-powering the Internet of Things, Science 353, 124 (2016).

[4] J. M. Perkel, The Internet of Things comes to the lab, Nature 542, 125 (2017).

[5] Y. Gil, M. Greaves, J. Hendler, and H. Hirsh, Amplify scientific discovery with artificial intelligence, Science 346, 171 (2014).

[6] Z. Ghahramani, Probabilistic machine learning and artificial intelligence, Nature 521, 452 (2015).

[7] J. M. Kahn and K.-P. Ho, A bottleneck for optical fibres, Nature 411, 1007 (2001).

[8] A. H. Atabaki, S. Moazeni, F. Pavanello, H. Gevorgyan, J. Notaros, L. Alloatti, M. T. Wade, C. Sun, S. A. Kruger, H. Meng, K. Al Qubaisi, I. Wang, B. Zhang, A. Khilo, C. V. Baiocco, M. A. Popović, V. M. Stojanović, and R. J. Ram, Integrating photonics with silicon nanoelectronics for the next generation of systems on a chip, Nature 556, 349 (2018).

[9] R. Hanson, L. P. Kouwenhoven, J. R. Petta, S. Tarucha, and L. M. K. Vandersypen, Spins in few-electron quantum dots, Rev. Mod. Phys. 79, 1217 (2007).

[10] R. J. Warburton, Single spins in self-assembled quantum dots, Nat. Mater. 12, 483 (2013).

[11] D. D. Awschalom, L. C. Bassett, A. S. Dzurak, E. L. $\mathrm{Hu}$, and J. R. Petta, Quantum spintronics: Engineering and manipulating atom-like spins in semiconductors, Science 339, 1174 (2013).

[12] C. Chappert, A. Fert, and F. N. Van Dau, The emergence of spin electronics in data storage, Nat. Mater. 6, 813 (2007).

[13] A. Fert, Nobel lecture: Origin, development, and future of spintronics, Rev. Mod. Phys. 80, 1517 (2008).

[14] R. Fiederling, M. Keim, G. Reuscher, W. Ossau, G. Schmidt, A. Waag, and L. W. Molenkamp, Injection and detection of a spin-polarized current in a light-emitting diode, Nature 402, 787 (1999).

[15] D. Basu, D. Saha, and P. Bhattacharya, Optical Polarization Modulation and Gain Anisotropy in an Electrically Injected Spin Laser, Phys. Rev. Lett. 102, 093904 (2009).

[16] S. Das Sarma, J. Fabian, X. Hu, and I. Zutić, Spin electronics and spin computation, Solid State Commun. 119, 207 (2001).

[17] I. Zutić, J. Fabian, and S. Das Sarma, Spin-Polarized Transport in Inhomogeneous Magnetic Semiconductors: Theory of Magnetic/nonmagnetic p-N Junctions, Phys. Rev. Lett. 88, 066603 (2002).

[18] A. Tackeuchi, Y. Nishikawa, and O. Wada, Roomtemperature electron spin dynamics in $\mathrm{GaAs} / \mathrm{AlGaAs}$ quantum wells, Appl. Phys. Lett. 68, 797 (1996). 
[19] R. S. Britton, T. Grevatt, A. Malinowski, R. T. Harley, P. Perozzo, A. R. Cameron, and A. Miller, Room temperature spin relaxation in GaAs/AlGaAs multiple quantum wells, Appl. Phys. Lett. 73, 2140 (1998).

[20] M. Sugawara and M. Usami, Handling the heat, Nat. Photonics 3, 30 (2009).

[21] M. Paillard, X. Marie, P. Renucci, T. Amand, A. Jbeli, and J. M. Gérard, Spin Relaxation Quenching in Semiconductor Quantum Dots, Phys. Rev. Lett. 86, 1634 (2001).

[22] P. Borri, W. Langbein, S. Schneider, U. Woggon, R. Sellin, D. Ouyang, and D. Bimberg, Ultralong Dephasing Time in InGaAs Quantum Dots, Phys. Rev. Lett. 87, 157401 (2001).

[23] J. M. Elzerman, R. Hanson, L. H. Willems van Beveren, B. Witkamp, L. M. K. Vandersypen, and L. P. Kouwenhoven, Single-shot read-out of an individual electron spin in a quantum dot, Nature 430, 431 (2004).

[24] M. Kroutvar, Y. Ducommun, D. Heiss, M. Bichler, D. Schuh, G. Abstreiter, and J. J. Finley, Optically programmable electron spin memory using semiconductor quantum dots, Nature 432, 81 (2004).

[25] A. I. Tartakovskii, J. Cahill, M. N. Makhonin, D. M. Whittaker, J.-P. R. Wells, A. M. Fox, D. J. Mowbray, M. S. Skolnick, K. M. Groom, M. J. Steer, and M. Hopkinson, Dynamics of Coherent and Incoherent Spin Polarizations in Ensembles of Quantum Dots, Phys. Rev. Lett. 93, 057401 (2004).

[26] A. Tackeuchi, R. Ohtsubo, K. Yamaguchi, M. Murayama, T. Kitamura, T. Kuroda, and T. Takagahara, Spin relaxation dynamics in highly uniform InAs quantum dots, Appl. Phys. Lett. 84, 3576 (2004).

[27] X. M. Dou, B. Q. Sun, D. S. Jiang, H. Q. Ni, and Z. C. Niu, Temperature dependence of electron-spin relaxation in a single InAs quantum dot at zero applied magnetic field, J. Appl. Phys. 111, 053524 (2012).

[28] S. Sato, S. Hiura, J. Takayama, and A. Murayama, Temperature dependence of inter-dot electron-spin transfer among laterally coupled excited states in high-density InGaAs quantum dots, J. Appl. Phys. 127, 043904 (2020).

[29] S. Sato, S. Hiura, J. Takayama, and A. Murayama, Suppression of thermally excited electron-spin relaxation in InGaAs quantum dots using $P$-doped capping layers toward enhanced room-temperature spin polarization, Appl. Phys. Lett. 116, 182401 (2020).

[30] Y. M. Park, Y. J. Park, K. M. Kim, J. C. Shin, J. D. Song, J. Il Lee, and K.-H. Yoo, Carrier dynamics in an InGaAs dotsin-a-well structure formed by atomic-layer epitaxy, Phys. Rev. B 70, 035322 (2004).

[31] Y. M. Park, Y. J. Park, K. M. Kim, J. D. Song, J. I. I. Lee, K.-H. Yoo, H. S. Kim, and C. G. Park, Interdiffusion and structural change in an InGaAs dots-in-a-well structure by rapid thermal annealing, J. Appl. Phys. 96, 5496 (2004).

[32] J. Beyer, I. A. Buyanova, S. Suraprapapich, C. W. Tu, and W. M. Chen, Strong room-temperature optical and spin polarization in InAs/GaAs quantum dot structures, Appl. Phys. Lett. 98, 203110 (2011).

[33] X. D. Mu, Y. J. Ding, B. S. Ooi, and M. Hopkinson, Investigation of carrier dynamics on InAs quantum dots embedded in InGaAs/GaAs quantum wells based on timeresolved pump and probe differential photoluminescence, Appl. Phys. Lett. 89, 181924 (2006).
[34] S. Oertel, J. Hübner, and M. Oestreich, High temperature electron spin relaxation in bulk GaAs, Appl. Phys. Lett. 93, 132112 (2008).

[35] See Supplemental Material at http://link.aps.org/supple mental/10.1103/PhysRevApplied.14.044011 for AFM analysis of QD structures, BF-STEM data, additional PL data, and three-dimensional quantum simulations.

[36] F. Meier and B. P. Zakharchenya, Optical Orientation (Elsevier, North-Holland, 1984).

[37] S. Cortez, O. Krebs, S. Laurent, M. Senes, X. Marie, P. Voisin, R. Ferreira, G. Bastard, J.-M. Gérard, and T. Amand, Optically Driven Spin Memory in $n$-Doped InAs-GaAs Quantum Dots, Phys. Rev. Lett. 89, 207401 (2002).

[38] D. J. Hilton and C. L. Tang, Optical Orientation and Femtosecond Relaxation of Spin-Polarized Holes in GaAs, Phys. Rev. Lett. 89, 146601 (2002).

[39] H. M. G. A. Tholen, J. S. Wildmann, A. Rastelli, R. Trotta, C. E. Pryor, E. Zallo, O. G. Schmidt, P. M. Koenraad, and A. Y. Silov, Strain-induced $g$-factor tuning in single InGaAs/GaAs quantum dots, Phys. Rev. B 94, 245301 (2016).

[40] A. Lenz, R. Timm, H. Eisele, C. Hennig, S. K. Becker, R. L. Sellin, U. W. Pohl, D. Bimberg, and M. Dähne, Reversed truncated cone composition distribution of $\mathrm{In}_{0} .8 \mathrm{Ga}_{0} .2 \mathrm{As}$ quantum dots overgrown by an $\operatorname{In}_{0} \cdot 1 \mathrm{Ga}_{0} \cdot 9 \mathrm{As}$ layer in a GaAs matrix, Appl. Phys. Lett. 81, 5150 (2002).

[41] S. Paul, J. B. Roy, and P. K. Basu, Empirical expressions for the alloy composition and temperature dependence of the band gap and intrinsic carrier density in $\mathrm{Ga}_{x} \mathrm{In}_{1-x} \mathrm{As}, \mathrm{J}$. Appl. Phys. 69, 827 (1991).

[42] L. Brusaferri, S. Sanguinetti, E. Grilli, M. Guzzi, A. Bignazzi, F. Bogani, L. Carraresi, M. Colocci, A. Bosacchi, P. Frigeri, and S. Franchi, Thermally activated carrier transfer and luminescence line shape in selforganized InAs quantum dots, Appl. Phys. Lett. 69, 3354 (1996).

[43] Z. M. Wang, Self-Assembled Quantum Dots (SpringerVerlag, New York, 2008).

[44] D. González, D. F. Reyes, A. D. Utrilla, T. Ben, V. Braza, A. Guzman, A. Hierro, and J. M. Ulloa, General route for the decomposition of InAs quantum dots during the capping process, Nanotechnology 27, 125703 (2016).

[45] N. Liu, J. Tersoff, O. Baklenov, A. L. Holmes, and C. K. Shih, Nonuniform Composition Profile in $\mathrm{In}_{0} .5 \mathrm{Ga}_{0} .5 \mathrm{As}$ Alloy Quantum Dots, Phys. Rev. Lett. 84, 334 (2000).

[46] S. Birner, T. Zibold, T. Andlauer, T. Kubis, M. Sabathil, A. Trellakis, and P. Vogl, nextnano: General purpose 3D simulations, IEEE Trans. Electron Devices 54, 2137 (2007).

[47] Z. Y. Xu, Z. D. Lu, X. P. Yang, Z. L. Yuan, B. Z. Zheng, J. Z. Xu, W. K. Ge, Y. Wang, J. Wang, and L. L. Chang, Carrier relaxation and thermal activation of localized excitons in self-organized inAs multilayers grown on gaAs substrates, Phys. Rev. B 54, 11528 (1996).

[48] V. K. Kalevich, M. Paillard, K. V. Kavokin, X. Marie, A. R. Kovsh, T. Amand, A. E. Zhukov, Y. G. Musikhin, V. M. Ustinov, E. Vanelle, and B. P. Zakharchenya, Spin redistribution due to Pauli blocking in quantum dots, Phys. Rev. B 64, 045309 (2001). 